Ann. Biol. anim. Bioch. Biophys., 1977, 17 (5 B), 851-863.

\title{
Evolution postnatale de la lipogenèse dans le tissu adipeux et le foie du mouton et du lapin
}

\author{
A. VÉZINHET, J. NOUGUÈS \\ avec la collaboration technique de E. BOUTHIER, Thérèse CHERY el Odette MOULJERAC \\ Station de Physiologie animale I.N.R.A. \\ E.N.S.A. Place Viala, 34060 Montpellier Cedex.
}

Summary. Postnatal evolution of lipogenesis in adipose tissue and liver of lamb and rabbit.

The lipogenic activity of adipose and hepatic tissues was measured in vitro using tissue from growing lambs and rabbits. Acetate and glucose were used as precursors of fatty acid synthesis. Lipogenesis was estimated in relation to animal age, various deposits and adipose tissue cellularity.

In all groups of growing lambs, the highest rate of fatty acid was found in adipose tissue ; lipogenic activity in the liver was low. Subcutaneous and omental fat deposits were more active than perirenal deposits in younger lambs. Acetate was the predominant carbon source for lipogenesis; glucose incorporation was negligible. Lipogenic activity was closely correlated with the number of adipose cells.

In rabbits, lipogenesis occurred mostly in the liver; however, there was some lipogenic activity in adipose tissue. Acetate was the predominant fatty acid precursor as compared to glucose. Adipose tissue from various body sites showed a marked difference in lipogenic rafe. In younger growing rabbits, the internal deposits of omental and perirenal fat had a higher rate of fatty acid synthesis than did the subcutaneous adipose sites. As in the lambs, lipogenic activity was closely correlated with adipose cell number.

\section{Introduction.}

La lipogenèse a été essentiellement étudiée chez le Rat, comme modèle des monogastriques et chez les bovins ef les ovins après sevrage comme modèles des ruminants On a pu ainsi montrer que le glucose est leprécurseur privilégié de la synthèse des acides gras chez les monogastriques (Winegrad, 1965 ; O'Hea et Leveille, 1969). Chez les ruminants, c'est l'acétate qui est préférentiellement utilisé (Hanson et Ballard, 1967) alors que le glucose ne représente qu'une source peu imporlante de carbones (Lindsay, 1970).

Chez le Mouton, étant donné l'importance au cours de la phase d'allaitement et de sevrage du développement de la masse adipeuse, il étaił particulièrement intéressant d'étudier la lipogenèse comparativement à ce qu'elle devient chez les animaux plus âgés. 
Une étude semblable a été réalisée chez le Lapin en tenant compte que cet animal peut utiliser les glucides membranaires des végéłaux grâce au processus adapłatif de la coprophagie et à la présence d'une population bactérienne active dans le coecum.

Pour cet ensemble de raisons nous avons, chez ces deux espèces, consideré les activités lipogéniques in vitro d'adipocytes isolés, issus de différentes localisations anatomiques et incubés en présence de glucose $€ \nmid$ (ou) d'acétate, nous les avons aussi comparées à celles obtenues à partir de coupes de foie. La participation à la lipogenèse se présente, en effet, sous des aspects variés. Chez les ruminants, quel que soit leur âge, sa contribution serait faible selon Ingle ef al. (1972a) chez les ovins, Hood (1972) chez les bovins. Chez le jeune lapin en croissance, le rôle du foie dans la lipogenèse serait deux fois plus important que celui du tissu adipeux, ce rapport se trouverait inversé chez le Lapin adulte (Leung et Bauman, 1975).

Nous avons enfin considéré l'influence du nombre ef de la taille des adipocytes sur l'activité de synthèse des lipides.

\section{Matériel et méthodes.}

La lipogenèse dans les dépôts adipeux, est exprimée en nanomoles de précurseurs incorporés, après $3 \mathrm{~h}$ d'incubation par rapport aux lipides et aux acides gras totaux contenus dans $10^{6}$ adipocytes. Dans le cas du foie, l'expression est faite par rapport aux lipides et aux acides gras totaux d'un gramme de tissu frais.

Dans nos résultats, nous avons tenté de dégager la part de chacun des deux précurseurs dans les synthèses des lipides et des acides gras. Par ailleurs, pour un même précurseur, nous avons évalué le pourcentage de ce qui était incorporé dans différentes fractions, lipides totaux, acides gras totaux et par différence, glycérol. Nous avöns en effet constaté que l'incorporation des précurseurs dans les lipides insaponifiables était négligeable.

Ce travail a été réalisé sur 44 agneaux mâles Mérinos d'Arles. Ils étaient répartis en cinq lots comprenant des animaux âgés de 10, 25, 50, 100 et 250 jours. Ils étaient toujours nourris ad libifum, au moment du prélèvement des échantillons de dépôts adipeux et de foie.

En ce qui concerne les lapins, nous avons utilisé 66 mâles Néozélandais. Ils étaient répartis en cinq lots, aux âges de 35, 50, 75, 100 et 140 jours. Un lol de lapins âgés de 300 à 600 jours a été rajouté pour l'étude de la lipogenèse dans le tissu adipeux, et un lot de lapins de 200 jours pour la lipogenèse dans le tissu hépatique. Les animaux étaient foujours nourris ad libitum lors du prélèvement des échantillons de tissus adipeux et de foie.

\section{Résultats.}

\section{Agneaux}

1) Comparaison de l'utilisation d'acéfate ef de glucose.

Les figures 1 à 3 montrent que les adipocyłes des différents dépôts étudiés et les coupes de foie, sont capables de convertir l'acétate et le glucose en lipides et en acides gras. Ils le font cependant à des niveaux très variables. 
Dans les dépôts adipeux (fig. 1 et 2 ) on observe une lipogenèse nettement supérieure à celle du foie, surtout quand c'est l'acétate qui est utilisé comme précurseur. Celui-ci constitue la source privilégiée pour la synthèse des lipides chez l'Agneau, quel que soit l'âge de l'animal ou le dépôt considéré. Cette contribution de l'acétate à la lipogenèse croît avec l'âge alors que celle du glucose diminue.
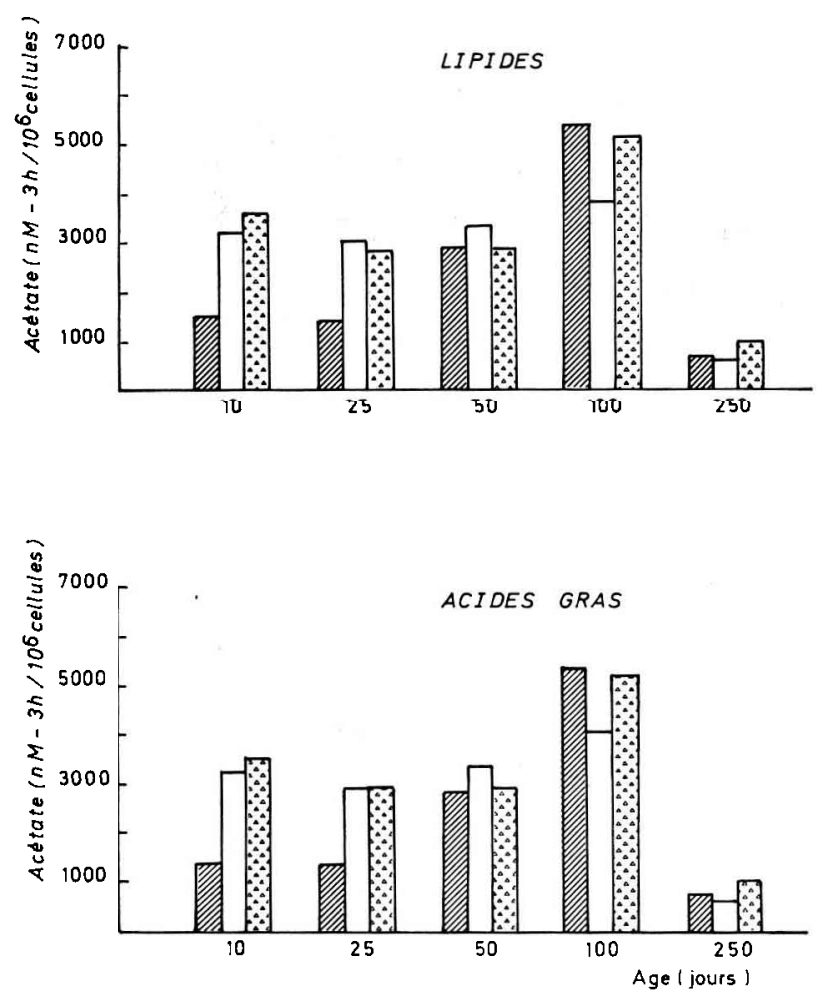

Dérirenal $\square$ omental $\quad$ :as sous-cutané

FIG. 1. - Comparaison de l'incorporation d'acétate dans les lipides ef les acides gras de $10^{6}$ adipocytes isolés provenant de différents dépôts chez des agneaux en croissance.

Dans les différents dépôts, la comparaison des incorporations d'acétate dans les lipides ef les acides gras totaux est intéressante à analyser. Elle révèle que la totalité de l'acétate qui est incorporée, l'est dans les acides gras totaux. II y a, en effet, une remarquable similitude entre l'incorporation de l'acétate dans les lipides et les acides gras de $10^{6}$ adipocytes, ce qui témoigne de l'absence de marquage dans le glycérol et les lipides insaponifiables.

La situation est différente pour le glucose dont une importante proportion est incorporée dans les lipides sous forme de glycérol. En outre, la contribution du glucose à la lipogenèse est faible, comparée à celle de l'acétate. 
Dans les coupes de foie (fig. 3) la synthèse des acides gras est faible depuis l'acétate et négligeable depuis le glucose, celui-ci est presque entièrement utilisé pour la synthèse du glycérol.
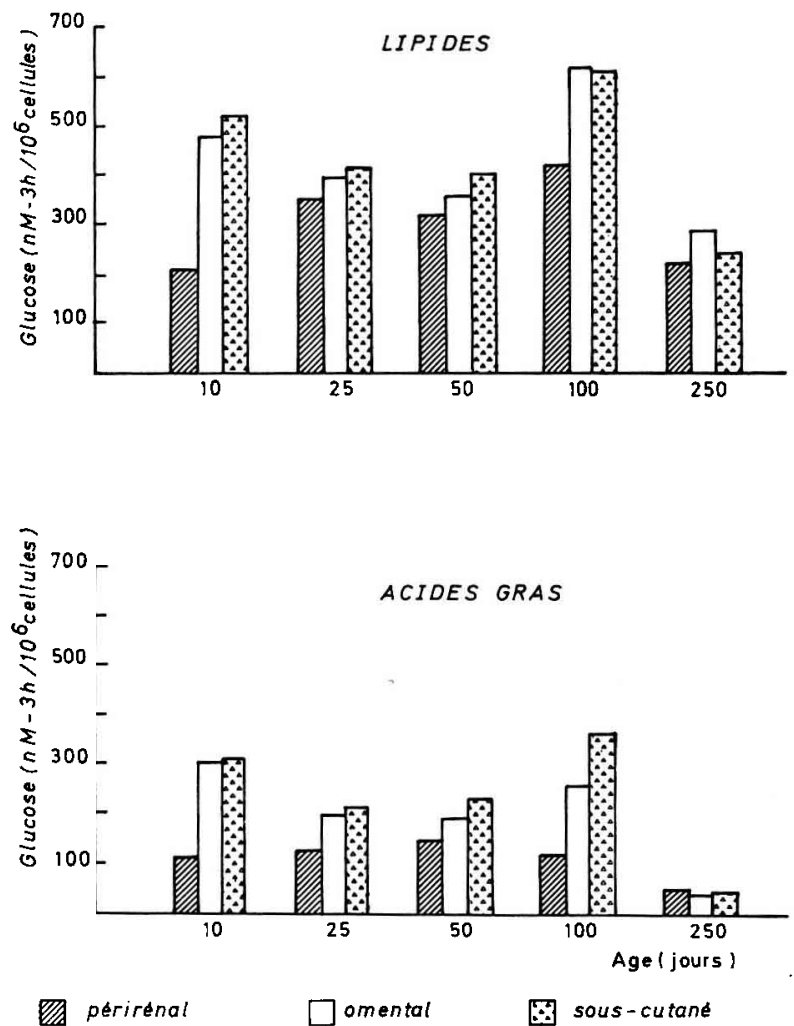

FIG. 2. - Comparaison de l'incorporation de glucose dans les lipides ef les acides gras de $10^{6}$ adipocytes isolés provenant de différents dépôts chez des agneaux en croissance.

2) Influence de l'âge des animaux.

Dans les dépôts adipeux : A dix jours pendant la phase postnatale précoce, d'alimentation exclusivement lactée, par rapport au glucose, l'acétate représente tout de suite le précurseur privilégié de la synthèse des lipides et des acides gras tołaux. II conservera tout au long de la période étudiée cette prééminence. Le glucose participe, néanmoins, à la synthèse des lipides tołaux dans une proportion non négligeable de 14 à 15 p. 100. Sa participation à la synthèse des acides gras totaux est moindre, comprise entre 8 et 10 p. 100. Le glucose aurait par contre un rôle important dans la fourniture de l' $\alpha$-glycérophosphate.

- A vingt cinq jours, la contribution du glucose à la synthèse des lipides reste inchangée, par contre son incorporation dans les acides gras tend à diminuer. 
- A cinquante et cent jours, le glucose joue un rôle moins important, que ce soit dans la synthèse des lipides $(7$ à 10 p. 100) ou celle des acides gras (4 p. 100 à 50 jours, 2 à 3 p. 100 à 100 jours).

- Enfin, à 250 jours, la lipogenèse est, de façon générale, réduite, que l'on utilise l'acétate ou le glucose comme précurseur des lipides. Tout se passe à cet âge comme si les adipocytes atteignaient un développement critique au-delà duquel leur aptitude à synthétiser des lipides serait fortement réduite.

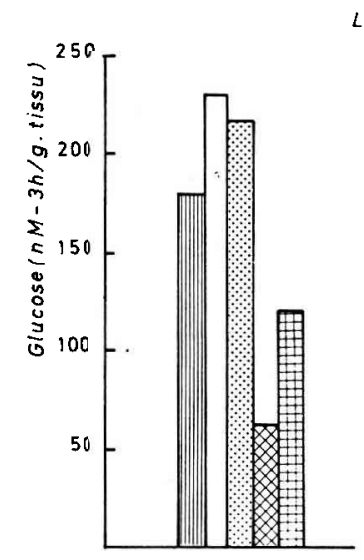

LIPIDES

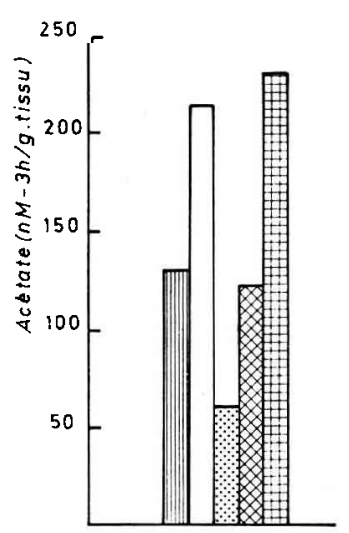

ACIDES GRAS
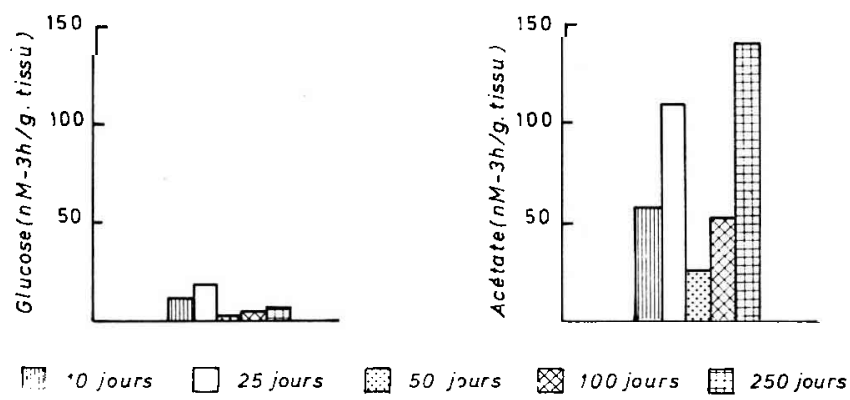

FIG. 3. - Comparaison de l'incorporation d'acétate ef de glucose dans les lipides ef les acides gras d'1 $\mathrm{g}$ de fissu hépatique chez des agneaux en croissance.

Dans le foie, la synthèse des acides gras est faible depuis l'acétate, négligeable depuis le glucose, celui-ci sert presque uniquement à la fourniture du glycérol. Dans le cas de l'acétate, nous avons observé qu'environ 25 p. 100 de la radioactivité incorporée dans les lipides tolaux ne se retrouvaił ni dans les acides gras ni dans les lipides insaponifiables. Bien qu'aucune voie métabolique connue ne permette d'envisager la synthèse du glycérol, à partir de l'acétate, cette possibilité pourrait exister par un transfert à travers le cycle des acides tricarboxyliques vers la voie de la gluconéogenèse ainsi que l'avait supposé Hood (1972). 
3) Influence du type de dépôt, du nombre et de la faille des adipocytes.

L'évolution de la lipogenèse dans le temps, par rapport à un nombre constant de cellules ( $10^{6}$ adipocytes), révèle que les dépôts omental et sous-cutané ont une activité de synthèse qui se maintient à un niveau sensiblement constant entre 10 et 100 jours. Les adipocytes du dépôt périrénal ont eux tendance à augmenter assez nettement leur activité durant la même période. La diminution de lipogenèse constatée à 250 jours, affecte tous les tissus.

\section{TABLEAU 1}

Corrélation ef paramètres de l'équation y $=a+b x$ dans laquelle $x$ représente le nombre de cellules présentes dans l'échantillon, y les nanomoles d'acétate incorporées dans les acides gras, a l'ordonnée à l'origine de la droife de régression dont b représente la pente. Période étudiée : 10 à 250 jours

\begin{tabular}{|c|c|c|c|c|c|}
\hline \multirow{2}{*}{$\begin{array}{l}\text { Dépôf étudié } \\
\text { (10 à } 250 \text { jours) }\end{array}$} & \multicolumn{5}{|c|}{$\begin{array}{c}\text { Nanomoles d'acétate incorporées dans les acides gras } \\
\text { totaux }-3 \mathrm{~h} / \text { nombre sde cellules } \times 10^{-3}\end{array}$} \\
\hline & $n$ & $r x y$ & b $y / x$ & Sb & $\mathbf{a}$ \\
\hline Périrénal $\ldots \ldots \ldots \ldots \ldots \ldots \ldots$ & 44 & $0,548 * * *$ & 1,255 & 0,289 & 115,59 \\
\hline 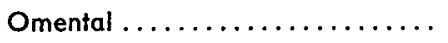 & 44 & $0,913 * * *$ & 3,069 & 0,211 & $-34,518$ \\
\hline Sous-cutané $\ldots \ldots \ldots \ldots \ldots \ldots$ & 44 & $0,835^{* * *}$ & 3,333 & 0,335 & $-41,362$ \\
\hline
\end{tabular}

r significatif à $P<1$ p. $100(* * *)$.

Ces observations sur les moyennes ont été complétées par une analyse en régression linéaire permettant d'apprécier les variations de l'activité lipogénique dans les échantillons incubés par rapport au nombre ef à la taille des adipocytes qu'ils contiennent.

Dans le cas de l'acétate, le tableau 1 montre que pour les trois dépôts étudiés (périrénal, omental et sous-cutané) l'incorporation d'acélate dans les acides gras est

\section{TABLEAU 2}

Corrélation ef paramètres de l'équation $y=a+b x$ dans laquelle $\times$ représente la faille des adipocytes exprimée par le diamètre moyen $(\mu \mathrm{m})$, y les nanomoles d'acéfafe incorporées dans les acides gras, a l'ordonnée à l'origine de la droite de régression dont b représente la pente. Période étudiée : 10 à 100 jours.

\begin{tabular}{|c|c|c|c|c|c|}
\hline \multirow{2}{*}{$\begin{array}{l}\text { Dépôt étudié } \\
\text { (10 à } 100 \text { jouts) }\end{array}$} & \multicolumn{5}{|c|}{$\begin{array}{l}\text { Nanomoles d'acétale incorporées dans les acides gras } \\
\text { totaux }-10^{6} \text { cellules }-3 \mathrm{~h} / \text { diamètre cellulaire moyen }\end{array}$} \\
\hline & $n$ & $r x y$ & b $y / x$ & $\mathrm{Sb}$ & a \\
\hline Périrénal $\ldots \ldots \ldots \ldots \ldots \ldots \ldots$ & 31 & $0,489 * * *$ & 65,815 & 14,581 & -1339 \\
\hline Omental $\ldots \ldots \ldots \ldots \ldots \ldots \ldots$ & 31 & $0,180 \mathrm{Ns}$ & 12,719 & 13,103 & 2529 \\
\hline Sous-cutané $\ldots \ldots \ldots \ldots \ldots$ & 31 & $0,244 \mathrm{Ns}$ & 29,216 & 21,564 & 1945 \\
\hline
\end{tabular}

r significatif à $\mathrm{P}<1$ p. $100(* * *)$, NS = non significatif. 
fortement corrélative du nombre d'adipocytes. La même analyse, appliquée au glucose, a montré que la corrélation qui lie l'activité lipogénique au nombre de cellules est, aussi, toujours hautement significative pour les trois dépôts.

En exprimant l'incorporation d'acétate dans les acides gras par rapport à la taille des adipocytes on note une absence de corrélation significative de l'incorporation d'acétate dans le cas des dépôts sous-cutané et omental. Seul le tissu périrénal présente une augmentation de sa capacité à synthétiser les acides gras corrélée avec l'accroissement de taille des adipocytes (tabl. 2).

\section{Lapins}

1) Comparaison de l'utilisation d'acétate et de glucose.

Les figures 4 à 6, montrent que les différents dépôts adipeux et le foie sont capables de convertir le glucose et l'acétate en lipides et en acides gras. L'acétate est, dans les deux tissus, le précurseur privilégié des acides gras mais,'à la différence del'Agneau, c'est le foie qui, en règle générale, joue le rôle essentiel dans cetfe synthèse.
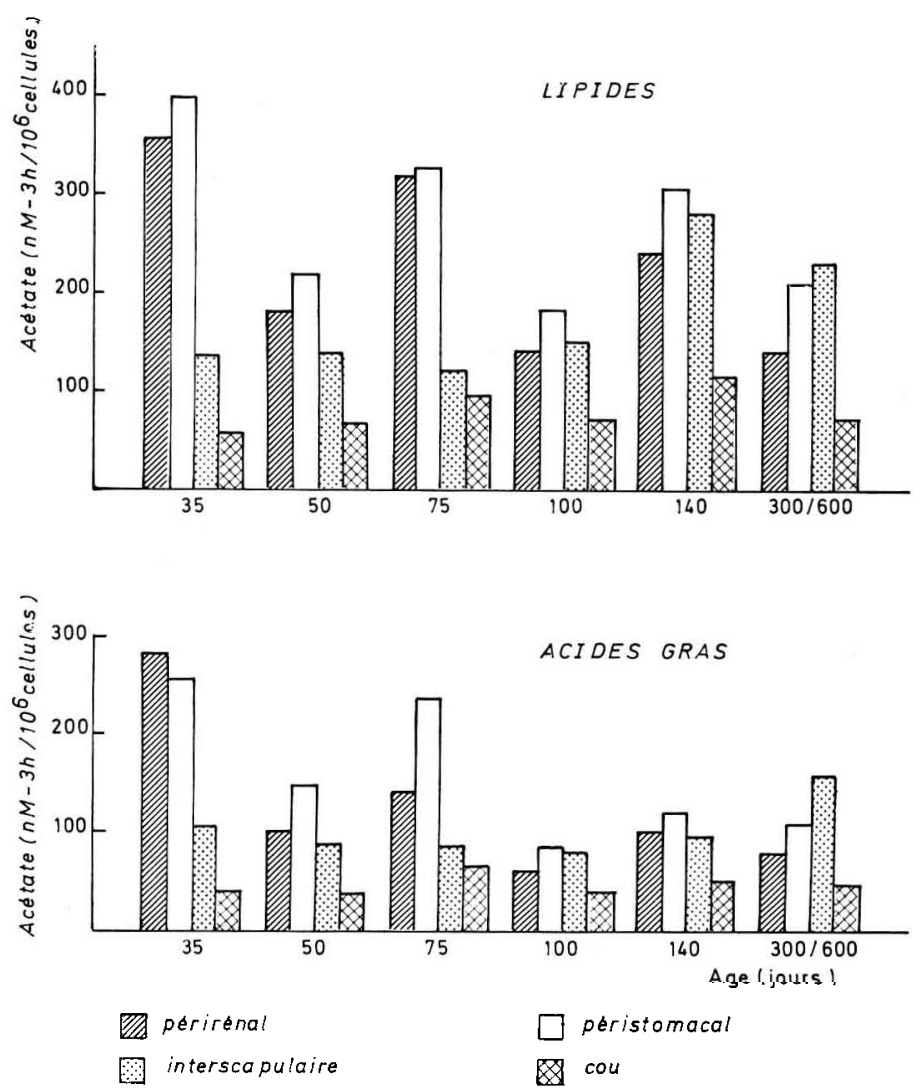

FIG. 4. - Comparaison de l'incorporation d'acétate dans les lipides ef les acides gros de $10^{6}$ adipocyies isolés provenant de différents dépôts chez des lapins en croissance. 
Dans les dépôts adipeux on ne retrouve dans les acides gras totaux que 50 p. 100 de l'acétate incorporé dans les lipides totaux. Comme on note une incorporation négligeable dans les lipides insaponifiables, il semblerait que 50 p. 100 d'acétate servent de précurseur à la fourniture de glycérol. Le résultat est à rapprocher de celui mis en évidence dans le foie de l'Agneau et suppose l'existence d'une voie métabolique méconnue.

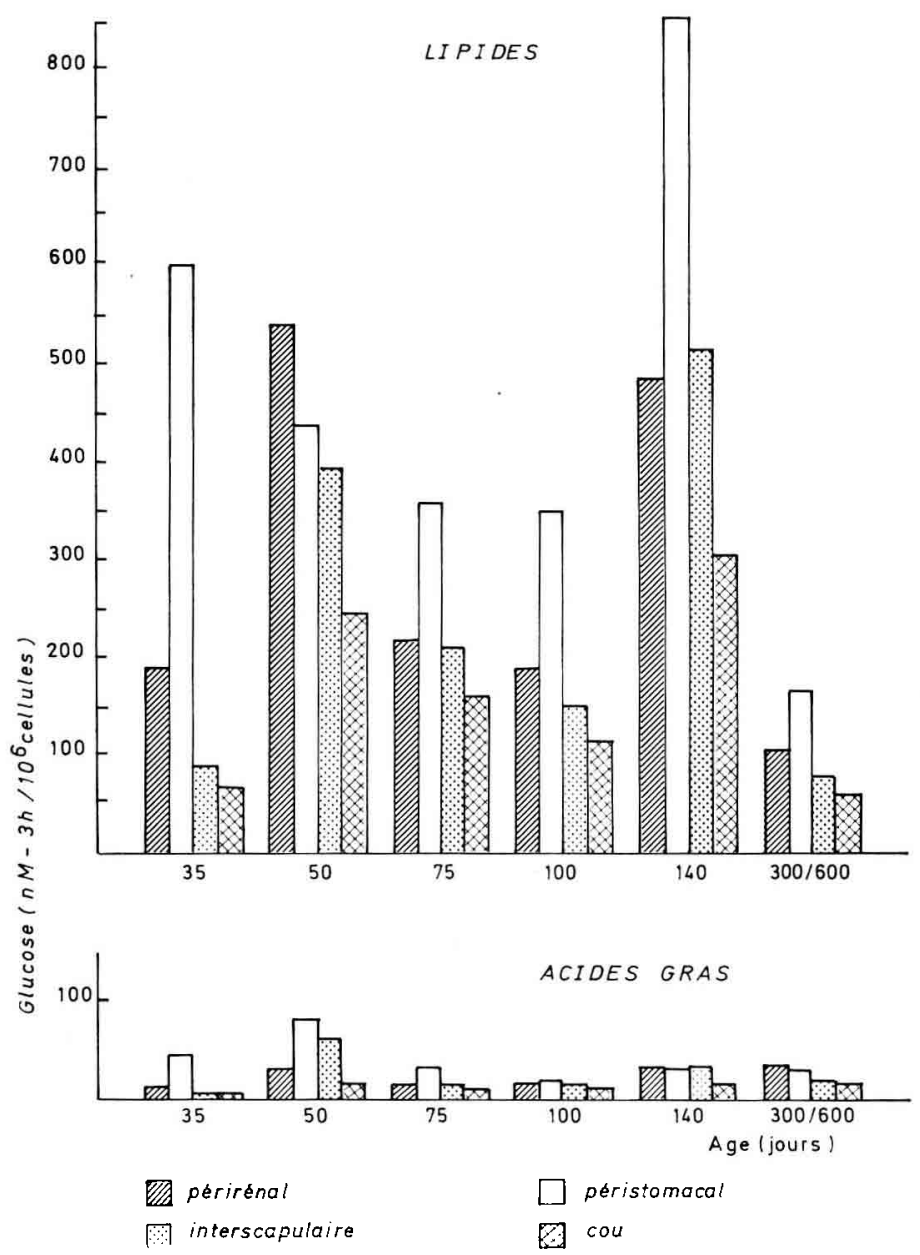

FIG. 5. - Comparaison de l'incorporation de glucose dans les lipides et les acides gras de $10^{6}$ adipocyles isolés provenant de différents dépôts chez des lapins en croissance.

L'incorporation du glucose est beaucoup plus faible que celle de l'acétate dans les acides gras, elle est cependant importante dans les lipides totaux; c'est dire qu'elle est essentiellement destinée à la fourniture d'a-glycérophosphate. 
Dans le foie après 35 jours, ef contrairement à ce qui a été observé chez l'Agneau, la lipogenèse est intense. Le tissu hépatique constitue, sans aucun doute, le site essentiel de cette fonction métabolique. L'acétate apparaît comme le précurseur presque exclusif de la synthèse des acides gras dans le foie. Le glucose est, par contre, faiblement utilisé.

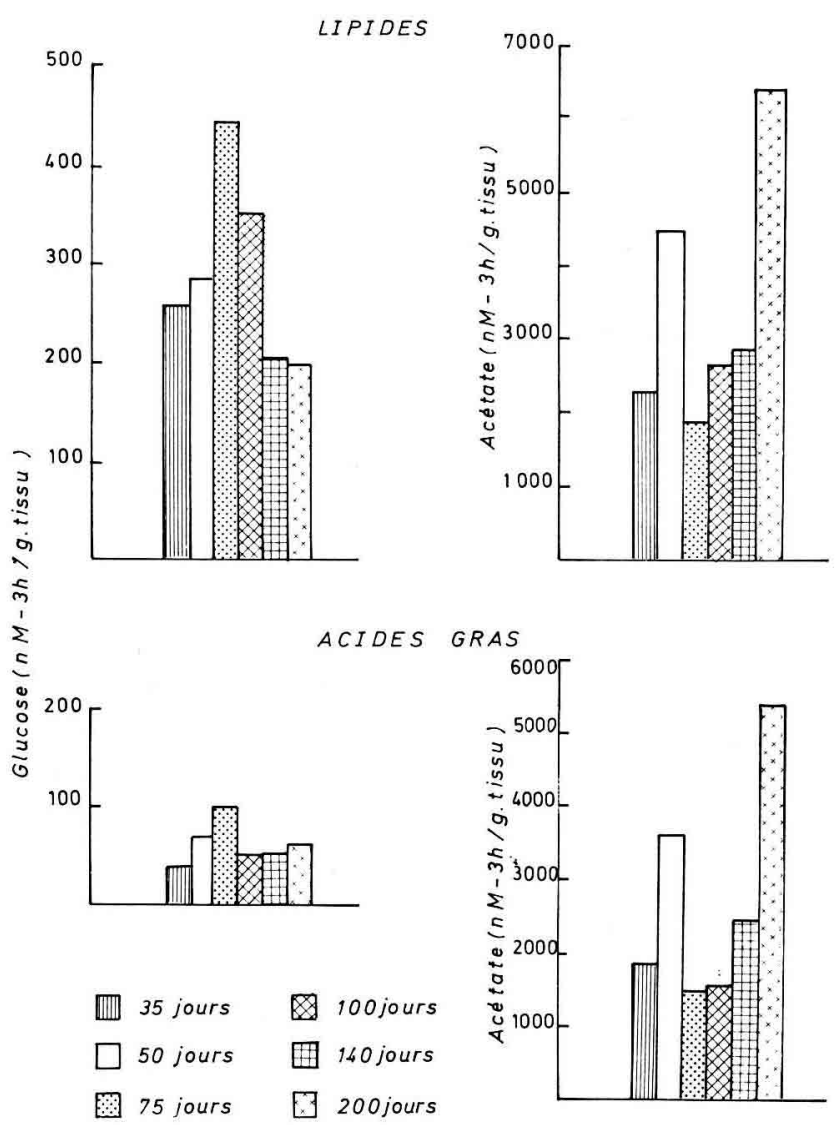

FIG. 6. - Comparaison de l'incorporation d'acétate et de glucose dans les lipides et les acides gras d'1 $\mathrm{g}$ de tissu hépatique chez des lapins en croissance.

2) Influence de l'âge.

Dans le tissu adipeux, à 35 jours on note une lipogenèse intense, notamment à partir de l'acétate qui fournit 80 à 95 p. 100 des acides gras formés, le glucose produisant le reste. A 50 jours, l'utilisation de l'acétate décroît en valeur absolue. Par rapport au glucose, sa participation à la synthèse des acides gras diminue aussi. A 75 jours, l'acétate maintient son rôle de précurseur essentiel, le glucose perd de l'importance. De 100 jours jusqu'au stade ultime des observations ( 600 jours) la lipogenèse à partir de l'acétate ef du glucose semble se maintenir à un niveau sensiblement constant. 
Dans le foie, la lipogenèse à 35 jours est déjà forte, à partir de l'acétate, bien qu'elle soit à cet âge concurrencée par celle qui s'effectue dans le tissu adipeux. A partir de 50 jours, le foie a une activité prédominante jusqu'à 140 jours. Enfin, à 200 jours, la moindre importance du foie, relativement au poids corporel, semble être compensée par une incorporation accrue de l'acétate dans les lipides ef les acides gras.

En ce qui concerne le glucose, comme nous l'avons déjà signalé, il esí peu utilisé pour la lipogenèse comparativement à l'acétate, cette situation n'évolue pas avec l'âge. Tout au long de la période étudiée, le glucose ne participe que pour une faible part (entre 1 et 6 p. 100) à la synthèse des acides gras contre 94 à 99 p. 100 à l'acétate.

3) Influence du type de dépôt, du nombre ef de la taille des adipocytes.

Les dépôts adipeux du Lapin se distinguent entre eux par des variations importantes de la taille de leurs adipocytes. L'augmentation du volume moyen des adipocytes des dépôts périrénal, péristomacal et interscapulaire est importante jusqu'à l'âge de 100 jours, puis elle se poursuit encore lentement jusqu'à environ 200 jours, où elle se stabilise. Le dépôt adipeux du cou se distingue tout au long de son développement par des adipocytes de faible taille. Compte tenu de ces caractéristiques histométriques, si on analyse l'activité lipogénique des différents dépôts adipeux on peut faire les remarques suivantes :

A trente cinq jours, la lipogenèse, à partir de l'acétate et (ou) du glucose dans les lipides et les acides gras totaux de $10^{6}$ adipocytes, est nettement plus faible dans les dépôts sous-cutanés (interscapulaire et du cou) que dans les tissus profonds (périrénal et surtout mésentérique). La faible activité du tissu du cou se maintient tout au long de la période étudiée, celle de l'interscapulaire est plus variable. Pour les dépôts périrénal el péristomacal, la meilleure utilisation de l'acétate semble se confirmer notamment pour le tissu mésentérique péristomacal au moins jusqu'à l'âge de 100 jours.

Les résultats sembleraient indiquer que les adipocytes de faible taille sont moins aptes, que les gros, à réaliser la lipogenèse.

TABLEAU 3

Corrélation ef paramètres de l'équation $y=a+b x$ dans laquelle $x$ représente le nombre de cellules présentes dans l'échantillon, y les nanomoles d'acétate incorporées dans les acides gras, a l'ordonnée d̀ l'origine de la droite de régression dont b représente la pente. Période étudiée : 35 à 600 jours.

\begin{tabular}{|c|c|c|c|c|c|}
\hline \multirow{2}{*}{$\begin{array}{l}\text { Dépôt étudié } \\
\text { (35 à } 600 \text { jours) }\end{array}$} & \multicolumn{5}{|c|}{$\begin{array}{c}\text { Nanomoles d'acétate incorporées dans les acides gras } \\
\text { totaux } 3 \mathrm{~h} / \text { nombre de cellules } \times 10^{-3}\end{array}$} \\
\hline & $n$ & $r x y$ & b $y / x$ & Sb & $a$ \\
\hline Périrénal .......... & 46 & $0,833 * * *$ & 0,321 & 0,032 & $-8,363$ \\
\hline Péristomacal $\ldots \ldots \ldots \ldots \ldots$ & 46 & $0,708 * * *$ & 0,224 & 0,034 & $-3,071$ \\
\hline Interscapulaire $\ldots \ldots \ldots \ldots \ldots$ & 46 & $0,730 * * *$ & 0,099 & 0,014 & $-0,267$ \\
\hline Cou $\ldots \ldots \ldots \ldots \ldots \ldots \ldots$ & 46 & $0,706 * * *$ & 0,051 & 0,008 & 0,164 \\
\hline
\end{tabular}

$r$ significatif à $P<1$ p. $100(* * *)$. 
Mais si on analyse pour chaque dépôt, la lipogenèse manifestée par $10^{6}$ adipocytes au cours des âges successifs, on s'aperçoit qu'elle varie peu de 35 à 600 jours. Ceci tend à donner au nombre d'adipocytes un rôle essentiel dans la lipogenèse.

Une analyse en régression linéaire confirme cette hypothèse. Le tableau 3 montre que, pour les quatre dépôts étudiés, la lipogenèse à partir de l'acétate est en étroite corrélation avec le nombre d'adipocytes présents dans le milieu. Nous avons, par contre, noté une absence totale de corrélation entre l'activité lipogénique et la taille des adipocytes entre 35 et 600 jours (tabl. 4) et ceci en dépit d'un important accroissement du diamètre moyen des adipocytes.

\section{TABLEAU 4}

Corrélation ef parapètres de l'équation $\mathrm{y}=\mathrm{a}+\mathrm{bx}$ dans laquelle $\mathrm{x}$ représente la taille des adipocyfes exprimée par le diamètre moyen $(\mu \mathrm{m})$, y les nanomoles d'acélate incorporées dans les acides gras, a l'ordonnée d̀ l'origine de la droite de régression dont b représente la pente. Période étudiée : 35 à 600 jours.

\begin{tabular}{|c|c|c|c|c|c|}
\hline \multirow{2}{*}{$\begin{array}{l}\text { Dépôt étudié } \\
\text { (35 à } 600 \text { jours) }\end{array}$} & \multicolumn{5}{|c|}{$\begin{array}{c}\text { Nanomoles d'acétate incorporées dans les acides gras totaux } \\
10^{6} \text { cellules }-3 \mathrm{~h} / \text { diamètre cellulaire moyen }\end{array}$} \\
\hline & $n$ & $r x y$ & b $y / x$ & Sb & a \\
\hline Périrénal................ & 46 & $-0,208$ & $-1,475$ & 0,694 & 264 \\
\hline Péristomacal $\ldots \ldots \ldots \ldots \ldots$ & 46 & 0,225 & $-1,273$ & 0,340 & 269 \\
\hline Interscapulaire............. & 46 & 0,174 & 0,450 & 0,393 & 55,3 \\
\hline Cou $\ldots . .$. & 46 & 0,150 & 0,249 & 0,248 & 30,0 \\
\hline
\end{tabular}

\section{Discussion.}

Nos résultats montrent que chez l'Agneau et le Lapin le foie et le tissu adipeux sont des sites essentiels de la lipogenèse. Leur importance relative varie cependant beaucoup d'une espèce à l'autre. L'Agneau en croissance assure l'essentiel de la lipogenèse à partir du tissu adipeux ce qui confirme et complète les résultats obtenus chez la Brebis par Ingle ef al. (1972a ef b). Nos conclusions sont aussi à rapprocher de celles obtenues chez les bovins par Ingle et al. (1972a), Hood et al. (1972), Pothoven et Beitz (1973). Chez le Lapin, le foie est un lieu important de synthèse des acides gras; ce résultal est conforme à ceux obtenus chez la Souris par Jansen et al. (1966) ; Muiruri et Leveille (1970); Hems et al. (1975). Chez les oiseaux, Goodridge et Ball (1967); O'Hea et Leveille (1969) et chez l'Homme, Shrago et al. (1971) ; attribuent aussi au tissu hépatique la capacité de réaliser la quasi-totalité de la fonction lipogénique, le tissu adipeux intervenant surtout comme lieu de stockage des acides gras préformés.

En ce qui concerre l'influence du précurseur dans la lipogenèse, nos résultats montrent l'utilisation préférentielle de l'acétate par rapport au glucose chez l'Agneau et le Lapin. Chez l'Agneau, nos données confirment le rôle essentiel joué par l'acétate dans la fourniture des carbones destinés à la synthèse des lipides, ce rôle est connu 
depuis les travaux de Hanson et Ballard (1967). Chez le Lapin il faut se rappeler ici que, depuis Rudman et Di Girolamo (1967), on considérait que le tissu adipeux du Lapin n'avait qu'une très faible capacité à synthétiser les acides gras. Cette observation avait été éfablie en utilisant le glucose comme précurseur. Nos travaux montrent clairement que si l'observation de Rudman ef Di Girolamo reste valable pour le glucose par contre, le tissu adipeux dispose d'une assez forte capacité à assurer la lipogenèse, si l'acétate est offert comme substrat. Saggerson (1974) et Smith (1975) ont aussi noté que le tissu adipeux du Lapin privilégiait l'acétate par rapport au glucose dans la synthèse des acides gras. Ainsi le système lipogénique du tissu adipeux du Lapin se rapprocherait de celui des ruminants. Ceci peut être relié au fait que le jeune lapereau consomme dès la naissance un lait particulièrement riche en lipides et très pauvre en sucre. II pourrait répondre à cette caractéristique, comme le faif le jeune rat vis-à-vis du lait de ratte qui possède des caractéristiques semblables (Ballard ef al. 1969). Par la suite, l'acétate pourrait être, physiologiquement, le précurseur essentiel des acides gras chez le Lapin en raison des fortes quantités d'acide acétique qui sont produites et absorbées dans le cæcum de cette espèce (Henning et Hird, 1972).

En ce qui concerne l'influence de la cellularité sur l'activité lipogéniqua des dépôts adipeux, il apparaît au vu de nos résultats, que la lipogenèse est essentiellement due au nombre d'adipocyłes, indépendamment de leur taille, cette observation limiterait le rôle de la cellule adipeuse à un phénomène essentiellement cytoplasmique ; la participation de la membrane cellulaire n'ayant que peu d'importance. Si on admet qu'en l'absence de toute stimulation hormonale, la membrane de l'adipocyle peut se laisser passivement traverser par des molécules comme le glucose ou l'acétate, cette hypothèse ne paraît pas à priori contradictoire avec ce que l'on sait du déroulement de la lipogenèse.

Enfin, les résultats relatifs à la lipogenèse des différents dépôts posent le problème de savoir si un dépôt adipeux particulier peut avoir un rôle prépondérant de synthèse des lipides par rapport à un autre dépôt. Dans cette hypothèse, il conviendrait d'en rechercher le déterminisme. II semble néanmoins nécessaire d'être très prudent dans la généralisation des observations, faiłes sur un type de dépôt, à la masse adipeuse totale.

Réunion Groupe Développement INRA/Productions animales Thiverval-Grignon, 14-15 ovril 1976.

Remerciements. - Ce travail a été réalisé avec l'aide de la D. G. R. S. T., contrat 7371654 et de l'I. N. R. A., ATP « Tissu adipeux » nº 65532.

\section{Références}

BALLARD F. J., HANSON R. W., KRONFELD D. S., 1969. Gluconeogenesis and lipogenesis in tissue from ruminant and non ruminant animals. Fed. Proc., 28, 213-231.

GOODRIDGE A. G., BALL. E. G., 1967. Lipogenesis in the pigeon : in vivo studies. Amer. J. Physiol., 213, 245-253.

HANSON R. W., BALLARD F. J., 1967. The relative significance of aceiate and glucose as precursors for lipid synthesis in liver and adipose tissue from ruminants. Biochem. J., 105, 529-536. 
HEMS D. A., RATH E. A., VERRINDER T. R., 1975. Fatty acid synthesis in liver and adipose tissue of normal and genetically obese (Ob/ob) mice during 24 hours cycle. Biochem. J., 150, 167-173.

HENNING S. J., HIRD F. J. R., 1972. Diurnal variations in the concentration of volatile fatty acids in the alimentary tracts of wild rabbits. Brit. J. Nutr., 27, 57-64.

HOOD R. L., 1972. Adipose tissue cellularity and lipogenic activity in porcine and bovine animals. Thèse Univ. Minnesota, pp. 298.

HOOD R. L., THOMPSON E. H., ALLEN C. E., 1972. The role of acetate, propionate and glucose as substrates for lipogenesis in bovine tissues. Int. J. Biochem., 3, 598-606.

INGLE D. L., BAUMAN D. E., GARRIGUS U. S., 1972a. Lipogenesis in the ruminant in vitro study of tissue sites, carbon source and reducing equivalent generation for fatty acid synthesis. J. Nufr., 102, 609-616.

INGLE D. L., BAUMAN D. E., GARRIGUS U. S., 1972b. Lipogenesis in the ruminant : in vivo site of fatty acid synthesis in sheep. J. Nutr., 102, 617-624.

JANSEN G. R., ZANETTI M. E., HUTCHINSON C. F., 1966. Studies on lipogenesis in vivo. Fatty acid and cholesterol synthesis during starvation and refeeding. Biochem. J., 101, 811-818.

LEUNG T. T., BAUMAN D. E., 1975. In vivo studies of the site of fatty acid synthesis in the rabbit. Int. J. Biochem., 6, 801-805.

LINDSAY D. B., 1970. Carbohydrate metabolism in ruminants. In Phillipson. A. T. Physiology of digestion and metabolism in the ruminant, Oriel Press, Newcastle, p. 438.

MUIRURI K. L., LEVEILLE G. A., 1970. In vitro fatty acid synthesis and enzyme activity in liver and adipose tissue of the mouse. Int. J. Biochem., 1, 254-260.

O'HEA E. K., LEVEILLE G. A., 1969. Significance of adipose tissue and liver as sites of fatty acid synthesis in the pig and the efficiency of utilization of various substrates for lipogenesis. $J$. Nutr., 99, 338-344.

POTHOVEN M. A., BEITZ D. C., 1973. Effect of adipose tissue site, animal weight, and long term fasting on lipogenesis in the bovine. J. Nutr., 103, 468-475.

RUDMAN D., Di GIROLAMO M., 1967. Comparative studies on the physiology of adipose tissue. Adv. Lipid. Res., 5, 35-117.

SAGGERSON E. D., 1974. Lipogenesis in rabbit isolated fat cells. Biochem. J., 142, 477-482.

SHRAGO E., GLENNON J. A., GORDON E. S., 1971. Comparative aspects of lipogenesis in mammalian tissues. Metabolism, 20, 54-62.

SMITH S., 1975. Lipogenesis in rabbit adipose tissue. J. Lipid Res., 16, 324-331.

WINEGRAD A. I., 1965. Studies on rat adipose tissue in vitro. Il. Effects of insulin on the metabolism of specifically labeled glucose. J. biol. Chem., 233, 273-281. 